\title{
Experimental vascular protective shield combined with vacuum sealing drainage prevents pressure on exposed vessels and accelerates wound repair
}

\author{
Weibin Du ${ }^{1 \#}$, Lihong $\mathrm{He}^{1 \#}$, Lixiang Wang ${ }^{1 \#}$, Huahui $\mathrm{Hu}^{1}$, Huateng Zhou ${ }^{1}$, Guanai Bao ${ }^{2,3}$, \\ Rongliang Chen ${ }^{1}$, Fuxiang Shen ${ }^{1}$, Renfu Quan ${ }^{1}$
}

${ }^{1}$ Research Institute of Orthopedics, the Affiliated Jiangnan Hospital of Zhejiang Chinese Medical University, Hangzhou, China; ${ }^{2}$ Department of Rehabilitation, Cancer Hospital of the University of Chinese Academy of Sciences (Zhejiang Cancer Hospital), Hangzhou, China; ${ }^{3}$ Institute of Cancer and Basic Medicine (IBMC), Chinese Academy of Sciences, Hangzhou, China

Contributions: (I) Conception and design: W Du, L He, L Wang, R Quan; (II) Administrative support: W Du, L Wang, R Quan; (III) Provision of study materials or patients: W Du, L Wang; (IV) Collection and assembly of data: L He, H Hu, H Zhou, G Bao; (V) Data analysis and interpretation: W Du, R Chen, F Shen; (VI) Manuscript writing: All authors; (VII) Final approval of manuscript: All authors.

\#These authors contributed equally to this work.

Correspondence to: Weibin Du, PhD; Prof. Renfu Quan. Research Institute of Orthopedics, the Affiliate Jiangnan Hospital of Zhejiang Chinese Medical University, Hangzhou 312001, China. Email: dwbbdm@163.com; quanrenfu@126.com.

Background: The sustained negative pressure created by vacuum sealing drainage (VSD) on exposed vascular wounds can result in blood vessel compression, embolism, or necrosis. The objective of this research was to explore the ability of an experimental vascular protective shield combined with VSD to protect exposed vessels of the lower limbs and accelerate wound repair.

Methods: (I) The vascular protective shield was prepared; (II) the material was subjected to acute toxicity and hemolysis tests; (III) and 30 New Zealand rabbits were divided into three groups: the control, VSD-only, and combined shield-VSD groups (with ten rabbits in each group). The wound-healing rate, myocardial function, wound histopathology, expression of angiogenesis markers, and exposed vascular compression of these three groups were compared on day 7.

Results: (I) The internal structure of the material was smooth; and (II) no toxicity or death was observed in mice of any group. The hemolysis rate in the combined shield-VSD group was very low. (III) The combined shield-VSD group showed a higher wound-healing rate, and higher levels of cluster of differentiation 31 (CD31), vascular endothelial growth factor (VEGF), and platelet-derived growth factor (PDGF), than the other groups $(\mathrm{P}<0.05)$, along with a better tissue healing rate. (IV) Left ventricular pressure fluctuations in the combined shield-VSD group were smaller than those in the VSD-only group $(\mathrm{P}<0.05)$. (V) Blood vessels in the control and combined shield-VSD group were not damaged, but were damaged in the VSD-only group.

Conclusions: The experimental vascular protective shield exhibited exceptional biosafety. The combination of this shield with VSD reduces influences on systolic and diastolic capacities of myocardium and avoids multiple compressions of exposed vessels, thus contributing to early vascularization of wounds and wound repair.

Keywords: Vascular protective shield; vacuum sealing drainage (VSD); exposed vessels; early vascularization; wound repair

^ORCID: 0000-0002-9698-4897. 
Submitted Mar 12, 2020. Accepted for publication Jul 27, 2020.

doi: 10.21037/apm-20-625

View this article at: http://dx.doi.org/10.21037/apm-20-625

\section{Introduction}

Skin is the first barrier between the human body and the external environment, and is a vital component of the immune system. It senses external stimuli, regulates body temperature and excretion of bodily fluids, prevents physical and chemical damage, and blocks the invasion of pathogenic microorganisms (1-3). Unfortunately, acute and chronic injuries, deep degree burns, diabetic foot ulcers, and pressure sores can result in skin defects and even exposure of tendons and blood vessels (4-6).

Vacuum sealing drainage (VSD) has been extensively applied in wound repair $(7,8)$. VSD accelerates wound repair via several mechanisms: (I) increase in local blood flow and improvement of blood microcirculation of the wound (9); (II) improvement of edema around the wound and decrease in vascular permeability (10); (III) prevention of bacterial growth and wound infection (11); (IV) creation of a sealed and moist healing environment, and acceleration of the growth of granulation tissue (12); and (V) stimulation of cell proliferation and differentiation (13). However, the sustained negative pressure created by VSD on exposed vascular wounds can result in blood vessel compression, embolism, or necrosis. It is therefore vital to expand the indications of VSD by protecting exposed vessels and accelerating wound repair. At present, the application of VSD on exposed vascular wounds, especially in soft tissue defects of lower limbs combined with vascular wounds, is not frequently attempted.

In this study, we explored the potential of our experimental vascular protective shield combined with VSD to protect the exposed vessels of injured lower limbs in New Zealand rabbits and accelerate wound repair. Our findings confirmed the biosafety of this shield. Moreover, the novel shield combined with VSD reduced influences on the systolic and diastolic capacities of the myocardium and markedly reduced compression of exposed vessels. Meanwhile, it accelerated early vascularization and wound repair. The vascular protective shield combined with VSD thus has considerable potential for novel therapeutic strategies in the clinical treatment of exposed vascular wounds.

We present the following article in accordance with the ARRIVE reporting checklist (available at http://dx.doi. org/10.21037/apm-20-625).

\section{Methods}

\section{Experimental animals}

A total of 20 Kunming mice (10 male and 10 female) were provided by Shanghai Lingchang Biotechnology Co., Ltd (Medical Experimental Animal Number: SCXK20180003). Thirty New Zealand rabbits were obtained from the Experimental Animal Center, Zhejiang Chinese Medical University. The purchase and feeding of the animals, along with other animal procedures, were following the guidelines for animal research from the National Institutes of Health and the Committee on Animal Research (14). The experimental protocol was set up according to the guidelines for animal research from the National Institutes of Health and the Committee on Animal Research and was approved by the Experimental Animal Ethics Committee of the Affiliated Jiangnan Hospital of Zhejiang Chinese Medical University (Xiaoshan Traditional Chinese Medical Hospital) (No. XSZYY-2017-020).

\section{Reagents and materials}

The reagents used included hematoxylin and eosin (HE) (Sigma, USA), RNA reagent kit (Generay, China), reverse transcription kit (Vazyme, China), anti-cluster of differentiation 31 (CD31) antibody (GeneTen, USA), anti-vascular endothelial growth factor (VEGF), and antiplatelet-derived growth factor (PDGF) antibodies (Bioss, China). The materials used included a VSD system and attachment materials (Daewoong, Korea), along with a biological signal acquisition and analysis system (Techman, Chengdu, China).

\section{Preparation of a novel experimental vascular protective shield and its observation by electron microscopy}

The tubing used for the experimental vascular protective shield was made from a sterile 4-mm diameter silicone rubber drainage tube from the VSD system. It was hemisected longitudinally to yield two vascular protective 
shields with 2-cm long semicircular profiles. To fix the vascular protective shield in place, small holes were made on each of the shields to allow needles to pass through, thereby connecting soft tissue on both sides. The shield was cut into transverse and longitudinal sections, and observed under a TM-1000 electron microscope.

\section{Toxicity testing of the vascular protective shield}

\section{Acute toxicity testing}

The Kunming mice were randomly assigned to an experimental group and a control group. They were intraperitoneally administered either $50 \mathrm{~mL} / \mathrm{kg}$ of material extract (experimental group) or normal saline (control group). They were housed in separated cages after administration. General condition, body mass, signs of toxicity, and number of deaths were assessed at 24,48 , and $72 \mathrm{~h}$ following administration.

\section{Hemolysis testing of the vascular protective shield material}

Three groups, with six tubes for each group, were used. Tubes of the experimental group (material extract), negative control group (normal saline), and positive control group (pure water) were prewarmed in a water bath at $37^{\circ} \mathrm{C}$ for $30 \mathrm{~min}$. Subsequently, $0.1 \mathrm{~mL}$ of diluted rat blood was added into each tube and kept warm for $1 \mathrm{~h}$. The mixture was centrifuged at 2,500 rpm for $5 \mathrm{~min}$, and the supernatant was subjected to $545 \mathrm{~nm}$ absorbance determination using a microplate reader. The hemolysis rate was calculated as follows: (absorbance in experimental group - absorbance in negative control group)/(absorbance in positive control group - absorbance in negative control group) $\times 100 \%$.

\section{Model of exposed vascular damage in New Zealand rabbits}

\section{Preparation of exposed vascular damage}

New Zealand rabbits were anesthetized by administration of $3 \%$ pentobarbital in the ear vein based on their body weight. After inguinal hairs were shaved and sterilized, a wound model with an exposed femoral artery was prepared (the wound size was $3 \mathrm{~cm} \times 4 \mathrm{~cm}$ ), followed by compression hemostasis. Intramuscular injection of 800,000 units of penicillin was postoperatively performed for 2 consecutive days to prevent wound infection.

\section{Grouping and intervention}

A total of 30 New Zealand rabbits with exposed vascular wound model were randomly classified into three groups: the control group, VSD-only group, and combined shieldVSD group (with 10 rabbits in each group). The control group received routine dressing change treatment, and the wound surface was washed with normal saline every day. After disinfection with povidone iodine, the wound was covered with sterile gauze dressing, and the fixed dressing was covered with biofilm. For the VSD-only group, wounds were covered with a VSD dressing of the corresponding size, and the wound edge was sutured around the dressing. After wiping the edge of the wound with alcohol, it was completely covered with biofilm. A suction cup was subsequently installed and connected to a negative pressure suction device. Negative pressure was adjusted to $-150 \mathrm{mmHg}$ and maintained for 7 days. For the combined shield-VSD group, the exposed femoral artery was first covered with a $2-\mathrm{cm}$ long vascular protective shield, and then operated according to the same procedure as the VSDonly group.

\section{Evaluations of outcomes following exposed vascular damage}

\section{Determination of wound area}

The wound area was measured using a Vernier caliper each day for 7 days following injury. The rate of change of the wound area was calculated as follows: (pre-treatment wound area - post-treatment wound area)/pre-treatment wound area $\times 100 \%$.

\section{Determination of systolic and diastolic capacities of myocardium}

On post-injury day 7 , the skin of the neck was cut to bluntly dissect muscles and expose the carotid artery. The catheter of the biological function experiment system was inserted into the left ventricle to examine the hemodynamics.

\section{HE staining}

Rabbit skin wound tissue was collected at post-injury day 7 and fixed in $4 \%$ paraformaldehyde for 3-5 days at room temperature. After dehydration and paraffin embedding, sections were cut at $4 \mu \mathrm{m}$ and stained with $\mathrm{HE}$ for histological examination.

\section{Immunofluorescence}

Marginal tissues of rabbit skin wound were collected at post-injury day 7 and prepared to yield $4 \mu \mathrm{m}$ sections as above. After antigen retrieval, sections were incubated with primary and secondary antibodies, stained with 
Table 1 Primers for polymerase chain reaction analysis

\begin{tabular}{llc}
\hline Gene & \multicolumn{1}{c}{ Primers sequences (5'-3') } & $\begin{array}{c}\text { Fragment } \\
\text { size (bp) }\end{array}$ \\
\hline CD31 & Forward: CCTGCTGAATGAGGTGGT & 106 \\
& Reverse: GTCCCCGTTCTCCTTGTAG & \\
VEGF & Forward: AAGACAGACGGGGTTGGA & 65 \\
& Reverse: GCAGGTCACTCACTTTGCC & \\
PDGF & Forward: AGCGTCCTCAGCCCCTAC & 152 \\
& Reverse: CGCAGCCAGATCCACAG & \\
Actin & Forward: TGCCGCCTGGAGAAAGC & 106 \\
& Reverse: CGACCTGGTCCTCGGTGTAG & \\
\hline
\end{tabular}

4',6-diamidino-2-phenylindole (DAPI) and coverslipped. CD31 staining was observed under a fluorescence microscope.

Quantitative reverse transcription polymerase chain reaction (RT-qPCR)

Primer design (CD31, VEGF, PDGF, and GAPDH) was completed by Sunny bio, Biotechnology Co., Ltd, Shanghai, using Premier 5.0. Total RNA in marginal tissues of rabbit skin wounds was extracted using TRIzol reagent and reverse transcribed into complementary DNA (cDNA). After polymerase chain reaction (PCR) amplification, relative levels were determined using the $2^{-\Delta \Delta \mathrm{Ct}}$ method. Primer sequences and product lengths are listed in Table 1.

\section{Western blot}

Total protein was extracted from marginal tissues of rabbit skin wound using RIPA, quantified by the BCA method, and subsequently loaded for electrophoresis. After transferring onto a polyvinylidene fluoride (PVDF) membrane, protein was blocked in $5 \%$ skim milk for $2 \mathrm{~h}$, incubated with primary antibodies at $4{ }^{\circ} \mathrm{C}$ overnight and secondary antibodies for $2 \mathrm{~h}$. Bands were exposed by enhanced chemiluminescence (ECL) and analyzed by Image-Pro Plus.

\section{Observation of vessel compression}

The degree of compression of exposed vessels on postinjury day 7 was assessed by observing pathological conditions of stenosis, deformity, and embolism.

\section{Statistical analysis}

Each experiment was repeated in triplicate. SPSS 20.0 was used for data analyses. Data are expressed as mean \pm standard deviation and analyzed by the Student's $t$-test. A P value $<0.05$ was considered statistically significant.

\section{Results}

\section{Diagram of vascular protective shield preparation and electron microscopy findings}

The vascular protective shields with semicircular profiles were prepared (Figure 1A). Electron microscopy findings of the semi-circular tubular hose showed that the internal structure of the material was smooth, dense, and evenly distributed. The material fit the vessel wall well. The longitudinal section showed a longitudinal layered structure, and the transverse section showed a more intense structure (Figure 1B,C).

\section{Non-toxicity of the vascular protective shield}

No toxic response or death was observed in mice of any group. No significant differences in body weight gain at 24, 48 , and $72 \mathrm{~h}$ were found in mice between the experimental group and control group $(\mathrm{P}>0.05)$. Thus, the vascular protective shield is apparently non-toxic (Figure 1D). The determined absorbance in the experimental group, negative control group, and positive control group was $0.0416 \pm 0.00051,0.0424 \pm 0.00073$, and $0.2419 \pm 0.00095$, respectively. The hemolysis rate in the experimental group was thus very low at $-0.434 \%$, and is clearly non-hemolytic.

\section{Preparation of exposed vascular damage and the usage of vascular protective shield}

An inguinal wound area (of $3 \mathrm{~cm} \times 4 \mathrm{~cm}$ ) was prepared in rabbits to expose the femoral artery. Soft tissue defects of the wound were observed (Figure $2 A$ ). The vascular protective shield covered the exposed vessels to prevent compression from VSD. Soft tissues on the both sides of the wound were tightly connected by passing a suture through from the holes on both sides of the shield (Figure 2B).

\section{Wound repair}

Wound area was narrowed down at post-injury day 7 in the control group. The formation of a small amount of granulation tissue at the wound margin and a local inflammatory response could be seen. In the VSD-only 

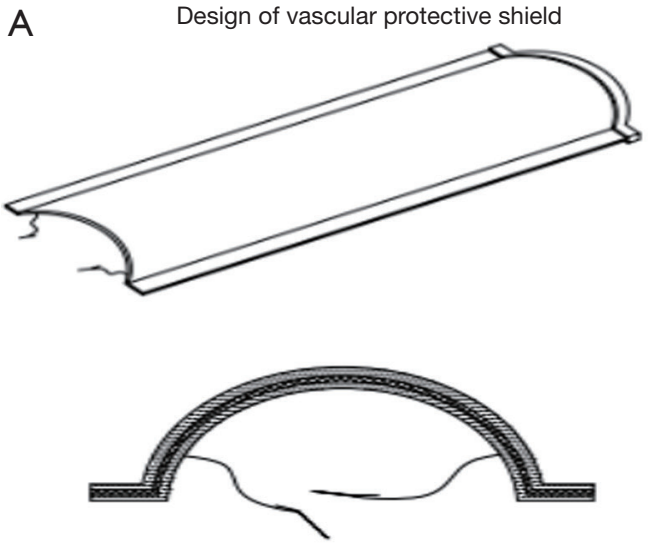

C

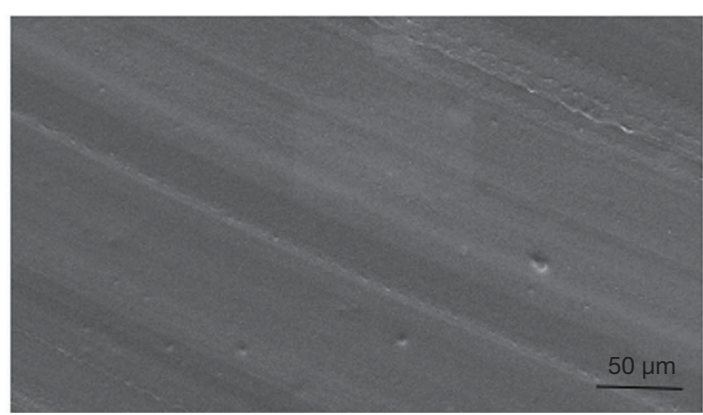

B
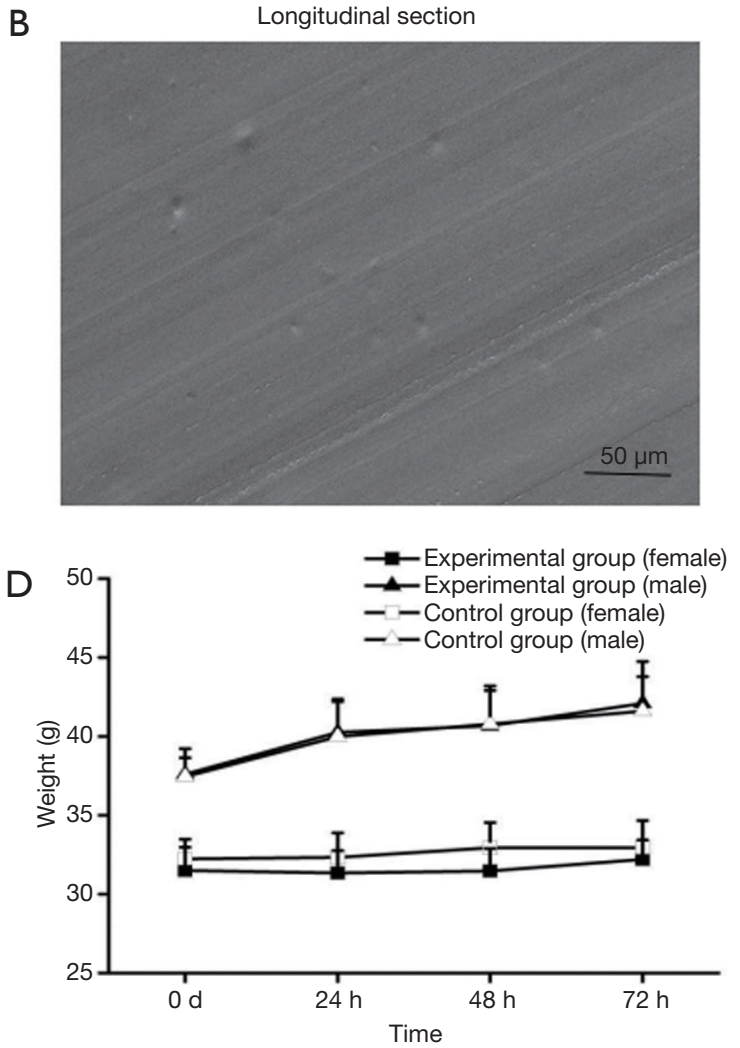

Figure 1 The drawing of vascular protective shield (A). The internal structure of cross section and longitudinal section was observed by SEM (B,C). The weight trends comparison of acute toxicity test in each group (D).

group, the wound area was significantly narrowed down. Moist wound margin and formation of a large amount of granulation tissue were observed, as well as buckling of vessels on the wound. Compared with the VSD-only group, wound narrowing and formation of granulation tissue were more pronounced in the combined shield-VSD group. Meanwhile, no obvious abnormalities in the exposed blood vessels of the wound were found (Figure 2C). Notably, the wound-healing rate at post-injury day 7 was higher in the VSD-only group $(49.82 \% \pm 5.72 \%)$ and the combined shield-VSD group $(57.47 \% \pm 4.70 \%)$ than in the control group $(35.26 \% \pm 5.23 \%)(\mathrm{P}<0.05)$. Furthermore, the woundhealing rate was significantly higher in the combined shieldVSD group compared with that of the VSD-only group $(\mathrm{P}<0.05)$ (Figure 2D).

\section{Changes of left ventricular pressure $+d p / d t$ max and $-d p /$ dtmax}

Fluctuations of $+d p / d t m a x$ and $-d p / d t m a x$ were significantly more pronounced in the combined shield-VSD group and VSD-only group at post-injury day 7 compared with the control group $(\mathrm{P}<0.05)$, which may be attributed to VSD. Nevertheless, fluctuations of $+\mathrm{dp} / \mathrm{dtmax}$ and $-\mathrm{dp} / \mathrm{dtmax}$ were smaller in the combined shield-VSD group compared with those of the VSD-only group $(\mathrm{P}<0.05)$, which may be attributed to the vascular protective shield (Figure $3 A, B$ ).

\section{HE staining and immunofluorescence results}

Histologically, tissue edema, inflammatory cell infiltration, and a small amount of granulation tissue were found in the control group. In the VSD-only group, abundant proliferation of fibroblasts, increased numbers of thickening collagen fibers (that were intense and disordered), and a small number of inflammatory cells were observed. Substantially more granulation tissue and collagen fibers were produced in the combined shield-VSD group. Meanwhile, epithelial cells and fibroblasts proliferated significantly (Figure 3C). Additionally, expression of CD31 


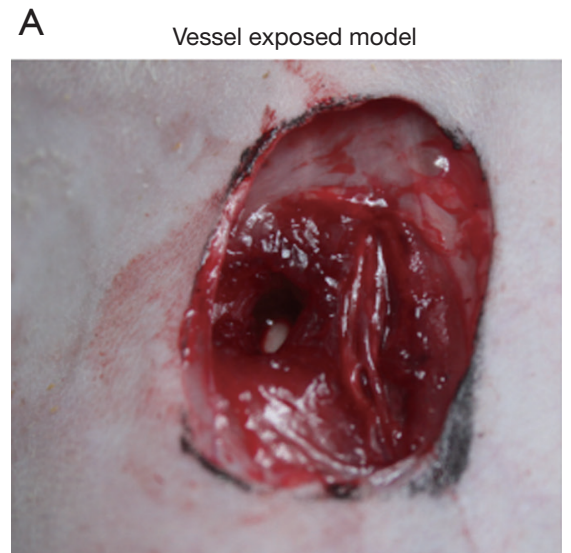

D

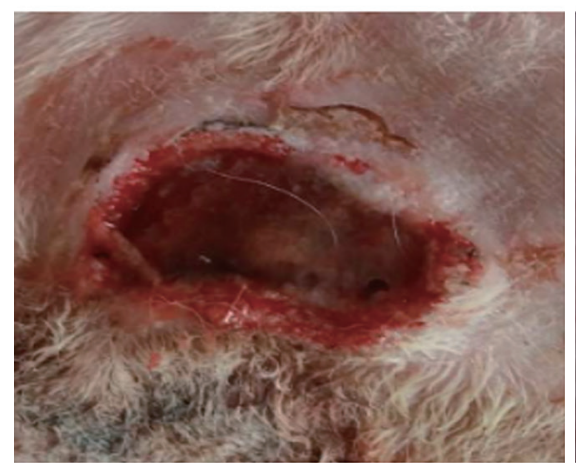

Model control group
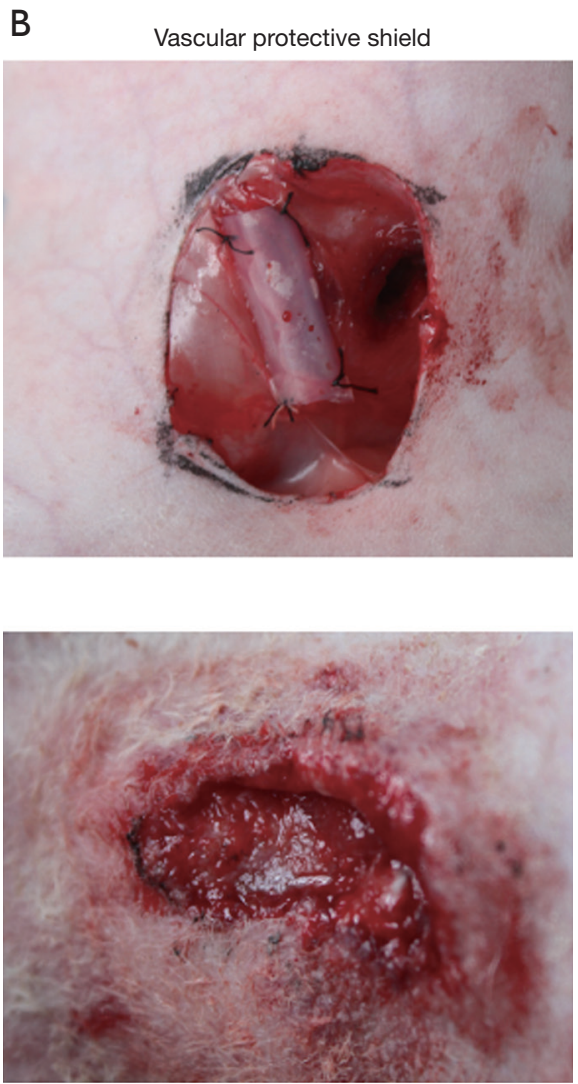

Single VSD group
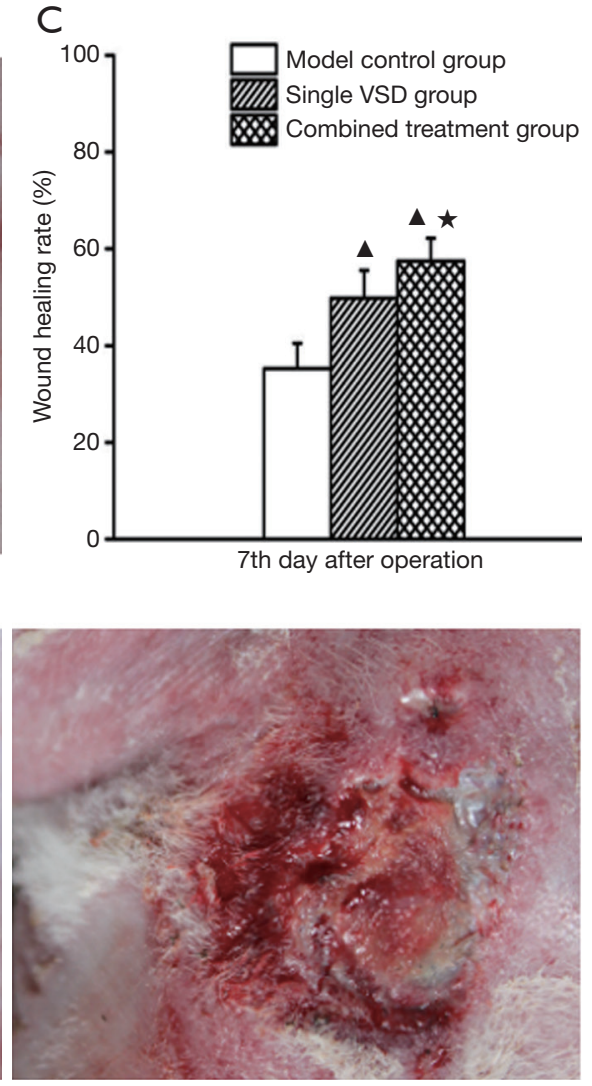

Combined treatment group

Figure 2 The preparation of bone and vessel exposed wound model (A). The vascular protective shield covers the exposed vessel (B). The wound healing rate and wound gross appearance change comparison in each group after 7 days (C,D). $\star, P<0.05 ; \boldsymbol{\Delta}, \mathrm{P}<0.01$.

was markedly higher in the combined shield-VSD group compared to the other two groups (Figure 3D).

\section{RT-qPCR results}

The mRNA levels of CD31, VEGF, and PDGF in the three groups were determined using the $2^{-\Delta \Delta C t}$ method. Levels of CD31, VEGF, and PDGF were higher in the combined shield-VSD group and VSD-only group compared with those in the control group $(\mathrm{P}<0.05)$, and were considerably higher in the combined shield-VSD group $(\mathrm{P}<0.05)$ (Figure 4A).

\section{Western blot results}

Protein levels of CD31, VEGF, and PDGF in the three groups were also examined. The Western blot results similarly revealed higher protein levels of CD31, VEGF, and PDGF in the combined shield-VSD group and VSD- only group compared with those in the control group $(\mathrm{P}<0.05)$, with this difference being significantly higher in the combined shield-VSD group $(\mathrm{P}<0.05)$ (Figure 4B,C).

\section{Vascular compression}

Compression of exposed vascular segments was observed on post-injury day 7 . Blood vessels were normal in the control group. However, vascular trauma and congestion were obvious in the VSD-only group, with the vascular walls becoming narrow and deformed, thereby posing a significant risk of local thrombosis. The vascular protective shield combined with VSD greatly protected the exposed vessels, the blood vessels were intact and unobstructed, and no vessel wall damage was observed (Figure 5).

\section{Discussion}

VSD is a physical negative pressure wound treatment that 

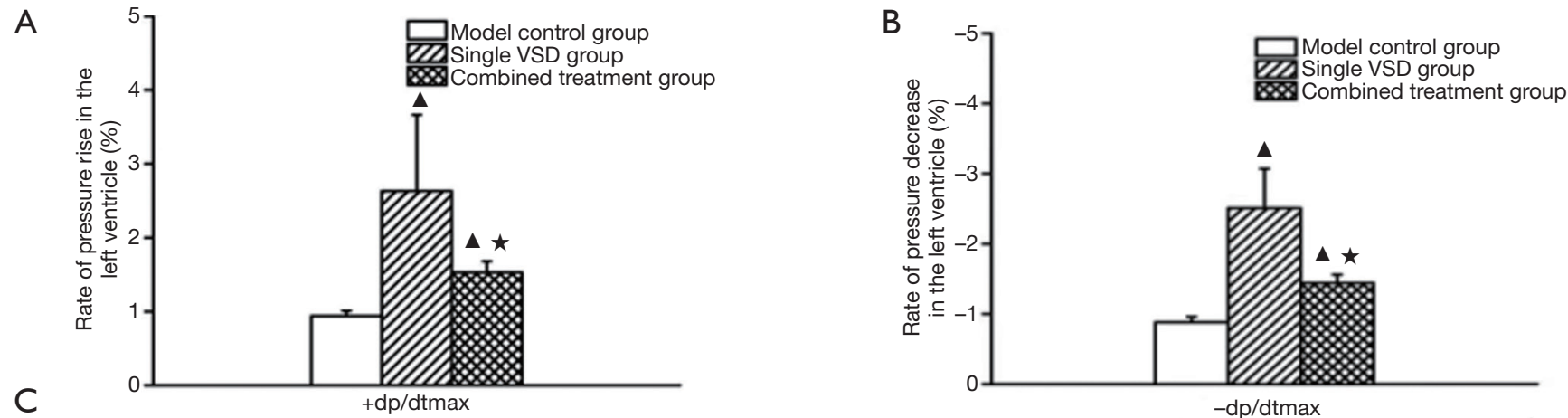

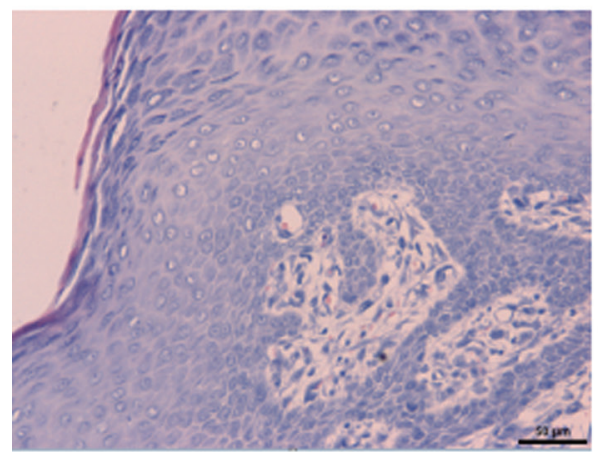

D

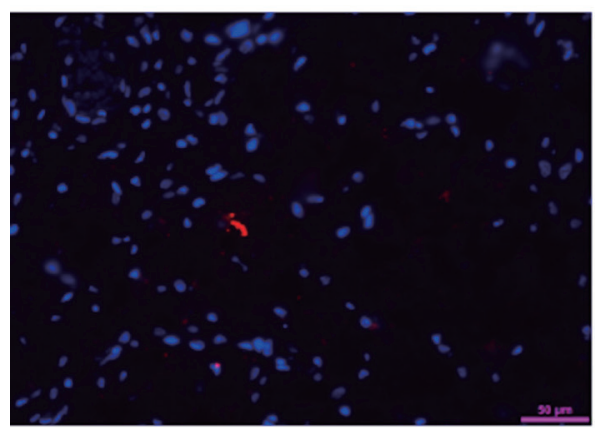

Model control group

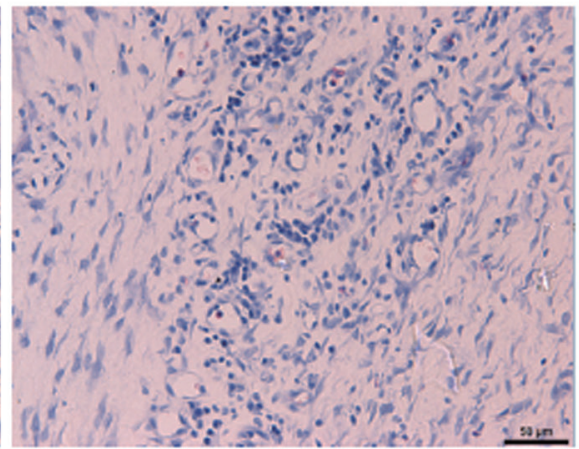

Single VSD group

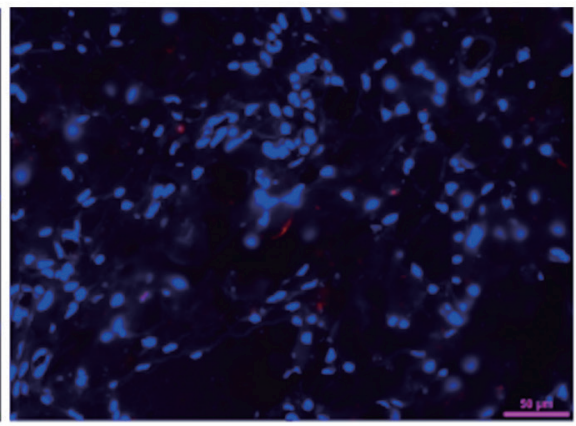

Single VSD group

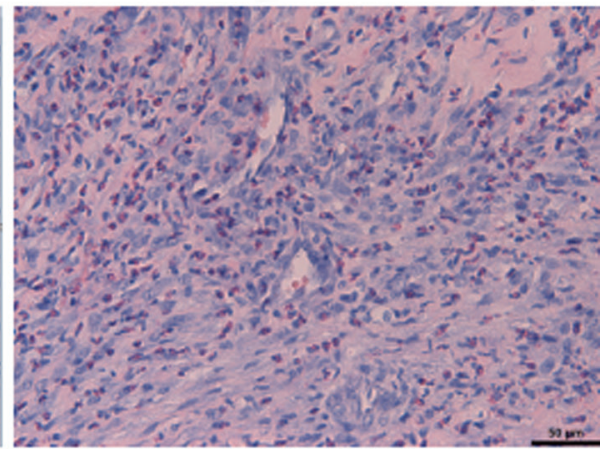

Combined treatment group

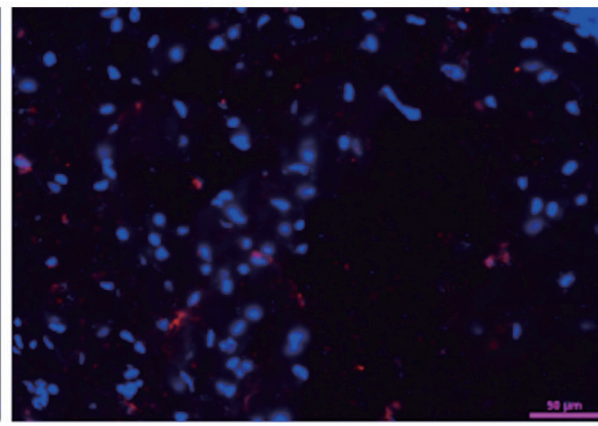

Combined treatment group

Figure 3 The $+d p / d t m a x$ and -dp/dtmax comparison of left ventricular pressure in each group (A,B). The histopathological changes of HE staining in each group (C). CD31 immunofluorescence in each group (D). $\star, P<0.05 ; \boldsymbol{\Lambda}, \mathrm{P}<0.01$.

has become popular over the past decade. It substantially enhances the efficacy of repair and treatment of various acute, chronic, and complex wounds through controllable negative pressure for drainage and adsorption (15-17). Indications of VSD are relatively broad, and include diabetic foot wounds (18), acne wounds (19), burn wounds (20), traumatic wounds (21), and infectious wounds (22). Wound healing is classified into three stages: the inflammatory phase, proliferative phase, and scar-remodeling phase. As a complex process that is relatively independent and influential, multiple factors are involved, such as angiogenesis and granulation tissue formation (23-26).
Abundant clinical evidence has proven the advantages of VSD in reducing wound edema, promoting formation of granulation tissue, and enhancing wound blood flow, thus triggering wound healing (27-29). DeFranzo et al. (30) proposed that VSD application significantly improves swelling of the lower limbs in patients with trauma and bone exposure after lower extremity injury, which provides a favorable condition for stage 2 skin grafting. Nevertheless, VSD-induced negative pressure may result in compression of exposed vessels, especially those on wounds with a hard base (i.e., bone surface). As a consequence, reduced local blood supply in the wound limits the timely removal of 

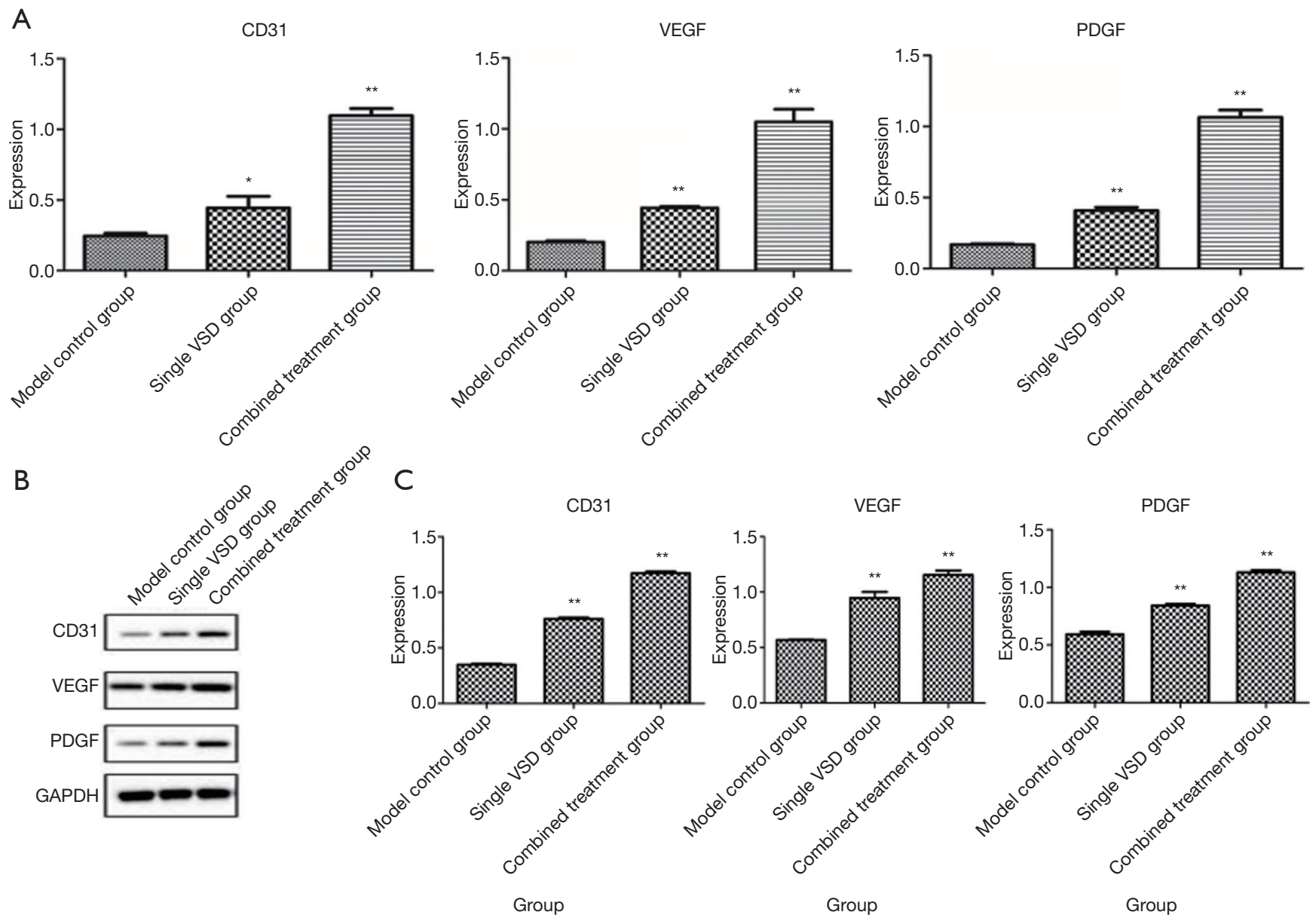

Figure 4 The mRNA levels comparison of RT-qPCR in each group (A). The protein expression and grey value comparison of Western blot in each group $(\mathrm{B}, \mathrm{C}) .{ }^{*}, \mathrm{P}<0.05 ;{ }^{* *}, \mathrm{P}<0.01$. RT-qPCR, quantitative reverse transcription polymerase chain reaction.

toxic substances and metabolites. Vascular occlusion or thrombosis may also occur. Eventually, vascular compression leads to ischemia and necrosis of wound skin, even in distal limbs. It is therefore necessary to avoid vascular compression on wounds at the time of VSD application.

In this experiment, the semi-circular vascular protective shield made of silicone rubber hose combined with VSD was applied to the treatment of soft tissue defects in New Zealand rabbits with vascular exposed wounds. Our findings demonstrated the following: (I) the vascular protective shield fit the vascular surface well. Electron microscopy showed that the internal structure of the material was smooth, dense. and evenly distributed. Moreover, the material used in the shield was non-toxic and non-hemolytic, further indicating its excellent biosafety. (II) Wound narrowing was significantly enhanced in the combined shield-VSD group at post-injury day 7 . Considerable granulation and new skin tissue were formed, and abnormalities were absent from the exposed vessels. The wound-healing rate was higher in the combined shield-VSD group and the VSDonly group than in the control group. Moreover, it was markedly higher in the combined shield-VSD group than in the VSD-only group. The above results demonstrate the obvious advantages of combined shield-VSD treatment in promoting wound repair compared to VSD-only treatment. (III) Fluctuations of $+d p / d t m a x$ and $-d p / d t m a x$ at post-injury day 7 were larger in the combined shieldVSD group and the VSD-only group than those of the control group, suggesting that VSD may influence blood pressure. Interestingly, fluctuations of $+\mathrm{dp} / \mathrm{dtmax}$ and $-\mathrm{dp} /$ dtmax were smaller in the combined shield-VSD group compared with those of VSD-only group. We believe that traumatic stress response and vascular compression resulted in systolic and diastolic changes of the rabbit myocardium. 


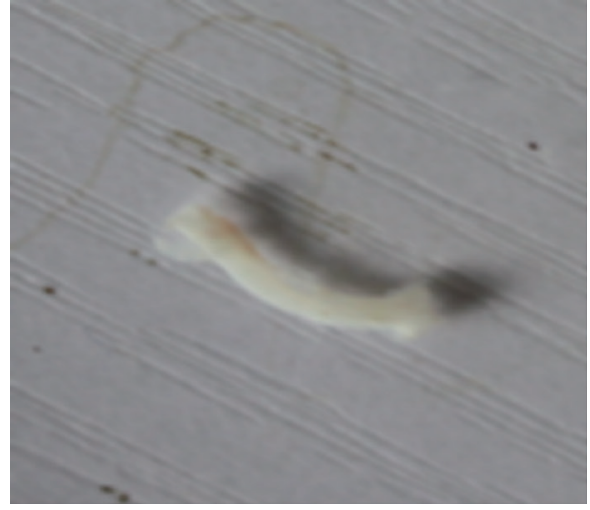

Model control group

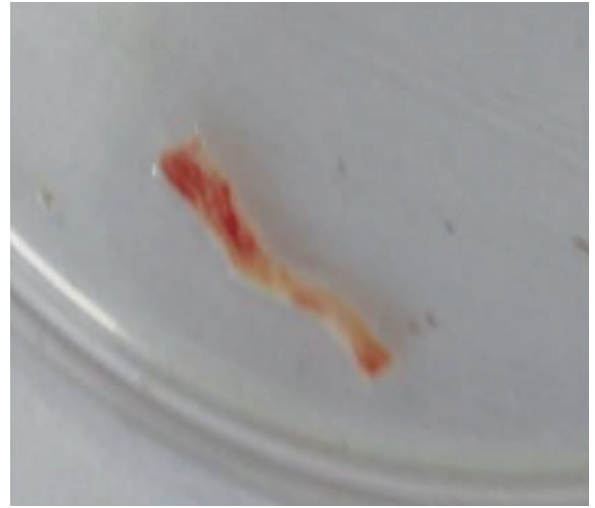

Single VSD group

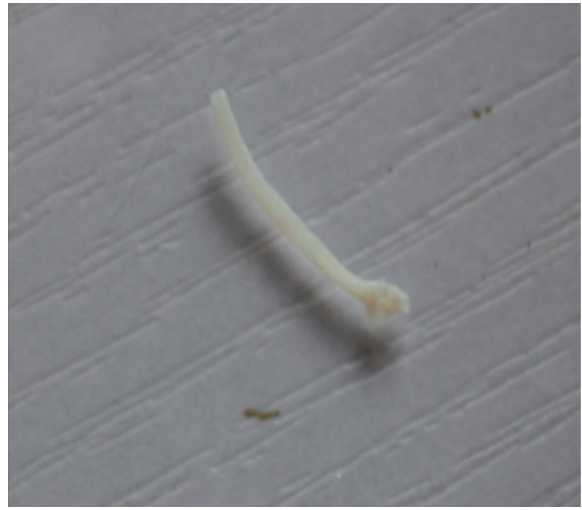

Combined treatment group

Figure 5 The gross appearance changes of exposed vessels after treatment in each group. VSD, vacuum sealing drainage.

Once the vascular protective shield was applied, decreased compression resulted in a lowered range of blood pressure fluctuation. (IV) Histopathology revealed substantially greater formation of granulation tissue and collagen, as well as pronounced proliferation of epithelial cells and fibroblasts in the combined shield-VSD group. Meanwhile, CD31 expression was higher in the combined shield-VSD group than in the other groups. It has been suggested that combined treatment not only protects exposed vessels, but also improves local blood circulation to stimulate angiogenesis and granulation formation. (V) RT-qPCR and Western blot results further confirmed the advantages of the vascular protective shield and VSD in stimulating early vascularization. Protection of exposed vessels guaranteed unobstructed local blood supply in the wound, while timely removal of toxic substances and metabolites upregulated vascular markers CD31, VEGF, and PDGF in the margin of the wound. As a result, capillary endothelial cells continued to proliferate and divide, thus accelerating wound repair. (VI) Vascular trauma and congestion were evident in the VSD-only group at post-injury day 7 , and the vascular walls became narrow and deformed. It has also been speculated that compression of the exposed arteries of the lower extremities inevitably disrupts the circulation of blood and wound repair. Furthermore, compression of the exposed veins can increase the risk of local thrombosis, and may even lead to pulmonary embolism. The vascular protective shield combined with VSD protected exposed vessels, blood vessels were intact and unobstructed, and no damage was found on the vessel wall. In summary, we found that the vascular protective shield greatly protected exposed vessels from compression and accelerated wound repair.
Some limitations of this study should be noted. (I) Materials of our experimental vascular protective shield were directly and easily collected from the VSD system. In future studies, material composition can be optimized through the use of $3 \mathrm{D}$ printing technology, thereby potentially improving biocompatibility, biosafety, and individualized application. (II) The short observation duration (7 days) and single negative pressure selection $(-150 \mathrm{mmHg})$ might have affected the dynamic evaluations on intervention period, negative pressure on the wound, and wound repair. Our forthcoming experiments will explore the influences of different observation durations and negative pressure selections on wound repair. (III) Research on early vascularization of the wound mainly focused on visible observation, pathological changes assessed by HE staining and immunofluorescence, and changes in the expression of vascular markers. In future, angiographic technologies should be applied for 3D visualization of the microvascular network, thus highlighting the importance of the vascular protective shield.

Collectively, this study confirmed that our experimental vascular protective shield combined with VSD effectively accelerated wound repair of exposed vessels, prevented compression of exposed vessels, and reduced influences on the systolic and diastolic capacities of myocardium. The combined therapy not only confirms the advantages of VSD in wound repair, but also demonstrates the benefits obtained by protecting the exposed vessels with the shield, which include an improvement of blood flow and supply of oxygen and nutrients. The experimental vascular protective shield combined with VSD is optimal for treating wounds with exposed vessels. 


\section{Acknowledgments}

Funding: This work was supported by National Natural Science Foundation of China (No. 81904053), the Plan Guide Project of Hangzhou Technology Department (No. 20171226Y93, 20171226Y96), the Plan Project of Hangzhou Health Science and Technology Department (No. 2018B030), and the Great Technology Capture Project of Hangzhou Xiaoshan District (No. 2017208, 2017214).

\section{Footnote}

Reporting Checklist: The authors have completed the ARRIVE reporting checklist. Available at http://dx.doi. org/10.21037/apm-20-625

Data Sharing Statement: Available at http://dx.doi. org/10.21037/apm-20-625

Conflicts of Interest: All authors have completed the ICMJE uniform disclosure form (available at http://dx.doi. org/10.21037/apm-20-625). The authors have no conflicts of interest to declare.

Ethical Statement: The authors are accountable for all aspects of the work in ensuring that questions related to the accuracy or integrity of any part of the work are appropriately investigated and resolved. The experimental protocol was set up according to the guidelines for animal research from the National Institutes of Health and the Committee on Animal Research and was approved by the Experimental Animal Ethics Committee of the Affiliated Jiangnan Hospital of Zhejiang Chinese Medical University (Xiaoshan Traditional Chinese Medical Hospital) (No. XSZYY-2017-020).

Open Access Statement: This is an Open Access article distributed in accordance with the Creative Commons Attribution-NonCommercial-NoDerivs 4.0 International License (CC BY-NC-ND 4.0), which permits the noncommercial replication and distribution of the article with the strict proviso that no changes or edits are made and the original work is properly cited (including links to both the formal publication through the relevant DOI and the license). See: https://creativecommons.org/licenses/by-nc-nd/4.0/.

\section{References}

1. Blechman AB, Cabell CE, Weinberger $\mathrm{CH}$, et al.
Aggressive Skin Cancers Occurring in Patients Treated With the Janus Kinase Inhibitor Ruxolitinib. J Drugs Dermatol 2017;16:508-11.

2. Vidal Yucha SE, Tamamoto KA, Kaplan DL. The importance of the neuro-immuno-cutaneous system on human skin equivalent design. Cell Prolif 2019;52:e12677.

3. Tsepkolenko A, Tsepkolenko V, Dash S, et al. The regenerative potential of skin and the immune system. Clin Cosmet Investig Dermatol 2019;12:519-32.

4. Hulsen J, Diederich R, Neumeister MW, et al. Integra dermal regenerative template application on exposed tendon. Hand (N Y) 2014;9:539-42.

5. Du WB, Wang L, Wu G, et al. Three-step sequential method for limb skin and soft tissue defect combined with bone or tendon exposed wound. Zhongguo Gu Shang 2019;32:742-5.

6. Chaput B, Bertheuil N, Grolleau JL, et al. Comparison of propeller perforator flap and venous supercharged propeller perforator flap in reconstruction of lower limb soft tissue defect: A prospective study. Microsurgery 2018;38:177-84.

7. $\mathrm{Li} Z, \mathrm{Wu} W$, Liu $\mathrm{S}$, et al. Effect of vacuum sealing drainage in dermatoplasty of large area of cutaneous defects. Int J Surg 2017;42:143-6.

8. Chen XJ, Liu S, Gao GZ, et al. Effects of vacuum sealing drainage on the treatment of cranial bone-exposed wounds in rabbits. Braz J Med Biol Res 2017;50:e5837.

9. Zhang M, Li Z, Wang J, et al. Effects of vacuum sealing drainage combined with irrigation of oxygen loaded fluid on chronic wounds in diabetic patients. Zhonghua Shao Shang Za Zhi 2014;30:116-23.

10. Liu J, Hu F, Tang J, et al. Homemade-device-induced negative pressure promotes wound healing more efficiently than VSD-induced positive pressure by regulating inflammation, proliferation and remodeling. Int J Mol Med 2017;39:879-88.

11. Tan L, Hou Z, Gao Y. Efficacy of combined treatment with vacuum sealing drainage and recombinant human epidermal growth factor for refractory wounds in the extremities and its effect on serum levels of IL-6, TNFand IL-2. Exp Ther Med 2018;15:288-94.

12. Yuan XG, Zhang X, Fu YX, et al. Sequential therapy with "vacuum sealing drainage-artificial dermis implantationthin partial thickness skin grafting" for deep and infected wound surfaces in children. Orthop Traumatol Surg Res 2016;102:369-73.

13. Hu C, Zhang T, Deng Z, et al. Study on the effect of vacuum sealing drainage on the repair process of rabbit 
sciatic nerve injury. Int J Neurosci 2015;125:855-60.

14. National Research Council (US) Committee for the Update of the Guide for the Care and Use of Laboratory Animals. The National Academies Collection: Reports funded by National Institutes of Health. Guide for the Care and Use of Laboratory Animals. Washington (DC): National Academies Press (US). 2011, National Academy of Sciences; 2011.

15. Lv Z, Yu L, Wang Q, et al. Dermal regeneration template and vacuum sealing drainage for treatment of traumatic degloving injuries of upper extremity in a single-stage procedure. ANZ J Surg 2019;89:950-4.

16. Shi B, Sun J, Cao Y, et al. Application of vacuum sealing drainage to the treatment of seawater-immersed blastinjury wounds. Int Wound J 2016;13:1198-205.

17. Wang R, Feng Y, Di B. Comparisons of negative pressure wound therapy and ultrasonic debridement for diabetic foot ulcers: a network meta-analysis. Int J Clin Exp Med 2015;8:12548-56.

18. Huang Q, Wang JT, Gu HC, et al. Comparison of Vacuum Sealing Drainage and Traditional Therapy for Treatment of Diabetic Foot Ulcers: A Meta-Analysis. J Foot Ankle Surg 2019;58:954-8.

19. Gao YB, Tong S, Pan F, et al. Myogenic elephant skin cream combined with Vacuum sealing drainage (VSD) for the treatment of bedsores. Zhongguo Gu Shang 2015;28:150-4.

20. Yuan DL, Zhao YH, Deng HT, et al. Application of selfmade vacuum sealing drainage device in postoperative fixation and drainage of abdominal pedicled flaps in 8 patients with deep burns of upper limbs. Zhonghua Shao Shang Za Zhi 2019;35:611-3.

21. Qu W, Ni S, Wang Z, et al. Severe open Lisfranc injuries:

Cite this article as: $\mathrm{Du} \mathrm{W}, \mathrm{He} \mathrm{L}$, Wang $\mathrm{L}, \mathrm{Hu} \mathrm{H}$, Zhou H, Bao G, Chen R, Shen F, Quan R. Experimental vascular protective shield combined with vacuum sealing drainage prevents pressure on exposed vessels and accelerates wound repair. Ann Palliat Med 2020;9(5):3059-3069. doi: 10.21037/apm$20-625$ one-stage operation through internal fixation associated with vacuum sealing drainage. J Orthop Surg Res 2016;11:134.

22. Chen X, Liu L, Nie W, et al. Vacuum Sealing Drainage Therapy for Refractory Infectious Wound on 16 Renal Transplant Recipients. Transplant Proc 2018;50:2479-84.

23. Sorg H, Tilkorn DJ, Hager S, et al. Skin Wound Healing: An Update on the Current Knowledge and Concepts. Eur Surg Res 2017;58:81-94.

24. Wang PH, Huang BS, Horng HC, et al. Wound healing. J Chin Med Assoc 2018;81:94-101.

25. Potter DA, Veitch D, Johnston GA. Scarring and wound healing. Br J Hosp Med (Lond) 2019;80:C166-C171.

26. Bi H, Li H, Zhang C, et al. Stromal vascular fraction promotes migration of fibroblasts and angiogenesis through regulation of extracellular matrix in the skin wound healing process. Stem Cell Res Ther 2019;10:302.

27. Liu X, Liang J, Zao J, et al. Vacuum Sealing Drainage Treatment Combined with Antibiotic-Impregnated Bone Cement for Treatment of Soft Tissue Defects and Infection. Med Sci Monit 2016;22:1959-65.

28. Wang J, Zhang H, Wang S. Application of vacuum sealing drainage in the treatment of internal fixation instrument exposure after early postoperative infection. Minerva Chir 2015;70:17-22.

29. Qu J, Yan R, Wang L, et al. Free dermatoplasty combined with vacuum sealing drainage for the treatment of largearea soft tissue defects accompanied by bone exposure in the lower leg. Exp Ther Med 2013;5:1375-80.

30. DeFranzo AJ, Aigenta LC, Marks MW, et al. The use of vacuum-assisted closure therapy for the treatment of lower-tremity wounds with exposed bone. Plast Reconstr Surg 2001;108:1184-91. 